\title{
Boundary Layer Trips for Low Reynolds Number Wind Tunnel Tests
}

\author{
A. Rona* \\ University of Leicester, Leicester LE1 7RH, United Kingdom \\ H. Soueid ${ }^{\dagger}$ \\ Contracted by Altran Sud Ouest at EDGVT, Airbus France, 316 route de Bayonne, Toulouse, France
}

The generation of a thick fully turbulent boundary layer is investigated in a lowspeed wind tunnel at a nominal zero pressure gradient over the Reynolds number range $0.145 \times 10^{6} \leq R e_{x} \leq 0.58 \times 10^{6}$. The wind tunnel floor natural boundary layer is laminar with thickness $\delta$ between $5.76 \mathrm{~mm}$ and $8.13 \mathrm{~mm}$. Different tripping devices are tested to trigger transition so to double the boundary layer thickness and provide a fully established turbulent velocity profile. Using a trip wire significantly increases $\delta$ but leads to an unsatisfactory velocity profile. Using a sandpaper strip slightly increases $\delta$ but keeps the boundary layer laminar. Using a strip of sharp-edged silicon granules doubles the boundary layer thickness that increases up to $20 \mathrm{~mm}$ and the mean velocity profiles are a good fit to the logarithmic law of the wall over the outer region of the boundary layer. The spectral decay of turbulent kinetic energy in this outer layer is exponential and close to $-5 / 3$, indicating turbulence equilibrium. This work is of practical interest to wind tunnel practitioners for generating equilibrium thick turbulent boundary layers at low Reynolds numbers.

\section{Nomenclature}

$B \quad$ Logarithmic law constant, $B=5.1$

$C \quad$ Sutherland law constant, $C=120 \mathrm{~K}$

$c_{1 \delta_{0}} \quad$ Weight of the boundary layer thickness error

$c_{1 H_{0}} \quad$ Weight of the boundary layer shape factor error

$f \quad$ Frequency

$f_{s} \quad$ Sampling frequency

$H \quad$ Boundary layer shape factor, $H=\delta^{*} / \theta$

$L \quad$ Characteristic length scale

$P S L \quad$ Constant bandwidth Power Spectral Level of kinetic energy

$<P S L>$ Ensemble averaged Power Spectral Levels of kinetic energy

$R e_{x} \quad$ Reynolds number based on the hot-wire position with respect to the tripping device

$R_{\theta} \quad$ Reynolds number based on momentum thickness

$T \quad$ Temperature

$T_{0} \quad$ Reference temperature, $T_{0}=291.15 \mathrm{~K}$

$t \quad$ Time

$u_{0} \quad$ Time-averaged velocity at $y_{0}$

$u_{\infty} \quad$ Free stream velocity of the wind tunnel

$u \quad$ Velocity component in the horizontal direction

$u^{+} \quad$ Normalized velocity, $u^{+}=u / u_{\tau}$

$u_{\tau} \quad$ Friction velocity, $u_{\tau}=\sqrt{\tau_{w} / \rho}$

$|\mathbf{u}| \quad$ Time-averaged velocity magnitude in the $(x, y)$ plane

$x_{p} \quad$ Horizontal distance between the test section leading-edge and the probe position

*Lecturer, Department of Engineering, AIAA Member.

${ }^{\dagger}$ Aircraft Performance Engineer, formerly Marie Curie Fellow at the University of Leicester. 


$\begin{array}{ll}x_{t} & \text { Horizontal distance between the test section leading-edge and tripping device position } \\ y & \text { Vertical distance with reference to the wind tunnel floor } \\ y_{0} & \text { Vertical position of the probe closest to the floor } \\ y^{+} & \text {Normalized vertical coordinate, } y^{+}=y u_{\tau} / \nu \\ \delta & \text { Boundary layer thickness } \\ \delta^{*} & \text { Boundary layer displacement thickness, } \delta^{*}=\int_{0}^{\delta}\left(1-u / u_{\infty}\right) d y \\ \theta & \text { Boundary layer momentum thickness, } \theta=\int_{0}^{\delta}\left(u / u_{\infty}\right)\left(1-u / u_{\infty}\right) d y \\ \kappa & \text { Von Kármán constant, } \kappa=0.41 \\ \nu & \text { Air kinematic viscosity } \\ \rho & \text { Density }\end{array}$

\section{Introduction}

Scaled up models are commonly used in wind tunnels to obtain spatially resolved measurements from geometries that are difficult to access, such as that of an automobile door seal cavity. ${ }^{1,2}$ To match the inflow Reynolds number at full-scale, these tests often require the generation of a fully turbulent inflow boundary layer at low speeds, the thickness of which is of the order of $20 \mathrm{~mm}$.

In a cavity flow, the momentum thickness of the boundary layer has a direct influence on the acoustic modes generated by the cavity and governs the growth of the shear layer across the cavity opening. ${ }^{3,4}$ Therefore, the ratio $L / \theta$, where $L$ is the cavity length and $\theta$ the incoming boundary layer momentum thickness, is one of the non-dimensional parameters to be matched in order to have a dynamic and geometric similarity between a real configuration and its equivalent wind-tunnel model.

It is difficult to generate a boundary layer inflow of prescribed thickness that is fully turbulent by simply relying on natural transition over a smooth flat plate. The thickness of a turbulent boundary layer $\delta$ is inversely proportional to the Reynolds number $R e_{x}$, for instance, in a $1 / 7^{t h}$ power law velocity profile, $\delta / x=$ $0.37 R e_{x}^{-0.2}$. Whilst the generation of a fully turbulent boundary layer drives towards a high Reynolds number, thickening a boundary layer points towards reducing $R e_{x}$. To overcome these conflicting requirements, it is common experimental practice to use a boundary layer leading edge trip. This allows to trigger the boundary layer transition to turbulence at a relatively low Reynolds number while increasing the boundary layer thickness by the thickness of the wake downstream of the trip. The practice of tripping boundary layers is well-established and relatively well documented in high Reynolds number flows. This paper contributes to the literature by investigating by experiment boundary layer tripping at low Reynolds numbers, with the dual objective of achieving a fully turbulent boundary layer, with well-developed turbulent statistics, and a pre-defined thickness, at a given location downstream of the trip.

This work tested three types of boundary layer trips: a wire, sand-paper at three different roughness grades, and polycarbonate granules, over the Reynolds number range $0.14 \times 10^{6} \leq R e_{x} \leq 0.58 \times 10^{6}$, where $R e_{x}=u_{\infty}\left(x_{p}-x_{t}\right) / \nu_{\infty}$ and $\left(x_{p}-x_{t}\right)$ is the streamwise distance between the trip and the boundary layer traverse. Section II details the wind tunnel test section and the instrumentation. Section III lists the matrix of running conditions. Section IV presents a summary of the boundary layer integral parameters ${ }^{5}$ $\left(\delta, \delta^{*}, \theta, H, u_{\tau}, R e_{\theta}\right)$ from all the configurations tested, to give the reader the overview of the working ranges of each tripping device. Section V examines in more detail the measurements from the 18 configurations tested, commenting on their repeatability, on the measured statistical uncertainties, and on the physical state and the statistics of the boundary layer that were obtained, against the ones of a fully developed turbulent boundary layer.

This paper wishes to be a useful aid to experimental aerodynamicists for selecting a boundary layer trip device in an application that requires a low velocity thick turbulent boundary layer inflow.

\section{Description of the experimental setup}

\section{II.A. Wind tunnel}

The experiments of sections IV and V have been carried out in the blow-down open-loop subsonic wind tunnel of fig. 1(a). The DRA tunnel maximum free stream velocity is approximately $40 \mathrm{~m} / \mathrm{s}$. A centrifugal fan driven by a variable speed three-phase motor pressurizes the upstream settling chamber, which is $0.65 \mathrm{~m} \times 1.1 \mathrm{~m} \times$ $1.45 \mathrm{~m}$ long. The settling chamber is in three parts. The inlet to the first part from the fan is $0.6 \mathrm{~m} \times 0.6 \mathrm{~m}$ 
in cross-section. It remains the same height and widens to $1.1 \mathrm{~m}$ over a length of $0.96 \mathrm{~m}$ where it joins the second part. The inlet to the second part is $1.1 \mathrm{~m}$ wide $\times 0.6 \mathrm{~m}$ high and increases in height to $1.45 \mathrm{~m}$ over a length of $1.05 \mathrm{~m}$ where it joins the third part. The third part has parallel sides and could be termed the proper settling chamber. It has a cross-section $1.1 \mathrm{~m}$ wide $\times 1.45 \mathrm{~m}$ high. Its length is $0.65 \mathrm{~m}$ before it joins the contraction. Honeycomb panels in the settling chamber reduce the free-stream turbulence level to about $0.35 \%$ of the free-stream velocity at $u_{\infty}=15.6 \mathrm{~m} / \mathrm{s}$. A $7.88: 1$ contraction connects the settling chamber to the test section. The contraction is $1.1 \mathrm{~m}$ wide $\times 1.45 \mathrm{~m}$ high at the inlet and $0.458 \mathrm{~m}$ square at the exit. The DRA tunnel test section is $1.18 \mathrm{~m}$ long and $0.458 \mathrm{~m}$ wide. The test section height increases from 0.458 $\mathrm{m}$ to $0.470 \mathrm{~m}$ along its length to compensate for the boundary layer displacement thickness growth on its walls, as sketched in figure 1. This geometry gives a quasi-vanishing streamwise pressure gradient. The test section terminates with a diffuser of area ratio $1: 2.25$ that discharges in the laboratory ambient air. The diffuser inlet is $0.458 \mathrm{~m} \times 0.470 \mathrm{~m}$ in cross-section and $0.675 \mathrm{~m}$ square at the exit. The diffuser is $2.49 \mathrm{~m}$ long, giving it an included diffusion angle of 5.2 degrees.
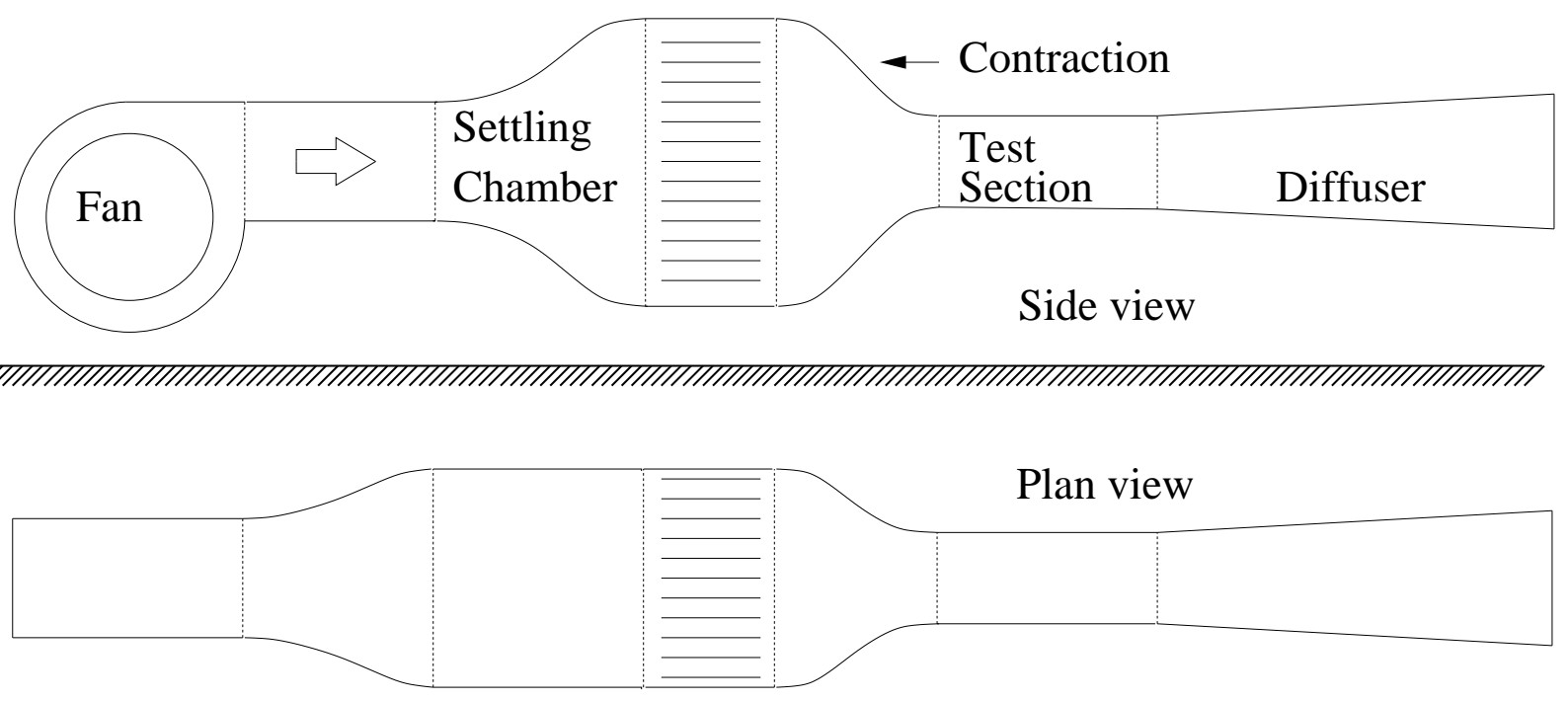

(a) Wind tunnel geometry.

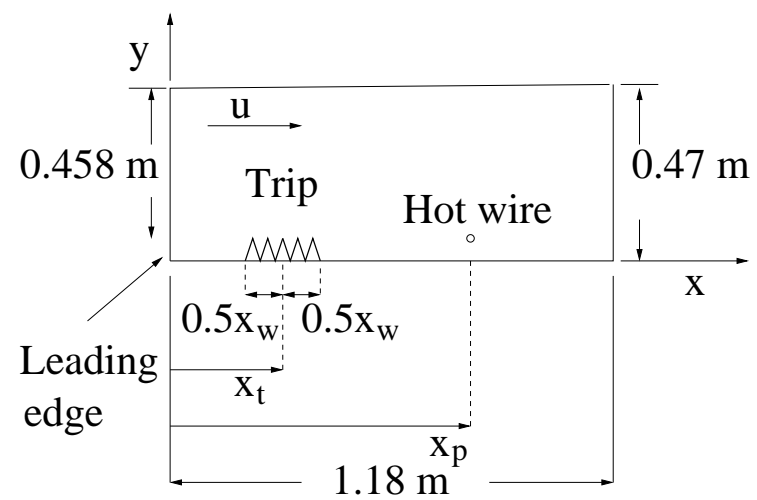

(b) Test section.

Figure 1. Sketch of the wind tunnel geometry.

\section{II.B. Flat plate model}

Measurements are performed on the wind tunnel test section floor under four configurations: (a) A natural boundary layer without any tripping device, (b) a trip wire of $3.5 \mathrm{~mm}$ diameter, (c) sandpaper strips of grades from P80 to P40, and (d) silicon granule strips with a characteristic granule size of $1.5 \mathrm{~mm}$.

In configuration d, the silicon granules are synthetic sand commonly used in fish tanks. They are sharp edged and their size was verified by sieving them through a coarse and then a fine sieve, to threshold their size about the nominal size of $1.5 \mathrm{~mm}$. The strip is made of double-sided tape on which the granules are 
in-laid.

The tripping devices are located at a distance $x_{t}$ from the test section leading edge, where $x_{t}$ identifies the streamwise centre of their planform area, as sketched in figure 1. The distance between the strips and the probe, $x_{p}-x_{t}$, in configurations $\mathrm{c}$ and $\mathrm{d}$ was modified to study its effect on the attainment of turbulent statistics that are characteristics of a fully turbulent boundary layer. The main reason for varying $x_{p}-x_{t}$ is that, at low Reynolds numbers, a surface perturbation of a laminar boundary layer induced by a roughness element, which is small compared to the boundary layer thickness, may be damped, maintaining a laminar flow over some distance downstream.

\section{II.C. Instrumentation}

The velocity in the boundary layer on the floor of the wind tunnel test section is measured using a hot-wire anemometer. A single $5 \mu \mathrm{m}$ diameter $1.25 \mathrm{~mm}$ wide $55 \mathrm{P} 15$ Dantec hot-wire probe is located at a distance $x_{p}$ from the leading edge of the test section. A traverse mechanism driven by a stepper motor is used to vary the vertical position of the probe, $y$, above the test section floor. 88 vertical positions are traversed between $y=y_{0}$ and $y=y_{0}+30 \mathrm{~mm}$ with an initial increment of $0.1 \mathrm{~mm}$. A Dantec constant temperature anemometer, model Dantec Streamline with a 90N10 frame and 90C10 constant temperature anemometer bridge, serial number 216, connects to the probe. The anemometer measures the voltage required to maintain the temperature of the hot-wire constant. At each vertical position $y$, the output voltage from the anemometer is recorded for $t=32$ seconds at a sampling frequency $f_{s}=512 \mathrm{~Hz}$. The record is then converted into velocity data using the voltage-velocity calibration curve of the probe. The calibration curve was obtained using a DISA type 55D41 calibration unit, by DISA Elektronik, DK, code 9058D0411, serial number 136. The mean velocity at each location $y$ is the average from the 32 seconds data. The initial position $y_{0}$ is estimated by achieving the contact between an identical broken probe and a conducting plate of a known thickness, placed on the wind tunnel floor. With this technique, the first measurement location was in the range $0<y_{0} \leq 0.5 \mathrm{~mm}$. The post-processing of the velocity profiles in the near-wall region presented in subsection V.D confirms this range.

\section{Test conditions}

The experiments were carried out at three nominal velocities of $3.9 \mathrm{~m} / \mathrm{s}, 7.8 \mathrm{~m} / \mathrm{s}$, and $15.6 \mathrm{~m} / \mathrm{s}$. These correspond to nominal Reynolds numbers $R e_{x}=0.145 \times 10^{6}, 0.29 \times 10^{6}$, and $0.58 \times 10^{6}$. During the tests, a temperature excursion of $18 \mathrm{~K}$ was recorded, leading to a variation of $5 \%$ in the molecular viscosity and thus in $R e_{x}$. By varying both $R e_{x}$ and the tripping devices, 18 configurations were tested, covering the range $0.14 \times 10^{6} \leq R e_{x} \leq 0.51 \times 10^{6}$, as detailed in table 1 . In table 1 , the actual test Reynolds numbers are stated and $\Delta x_{w}$ is the streamwise width of each tripping device.

\section{Effect of the tripping devices}

\section{IV.A. Integral boundary layer parameters}

The data acquired from each of the 18 configurations of table 1 are post-processed using in-house software written in Matlab. Starting from the set of the temporal voltage data, the average is computed and the mean voltage is transformed into the correspondent mean velocity based on the calibration curve of the hot-wire probe. This gives the mean streamwise velocity at each vertical position $y$. These vertical positions are determined from the stepper motor count record of the hot wire traverse, the lead screw pitch and the traverse starting position $y_{0}$. A $0.5 \mathrm{~mm}$ uncertainty in $y_{0}$ leads to a vertical shift of the velocity profile that is compensated for by the post-processing. Specifically, an iterative technique is adopted to compute accurately $y_{0}$ that also determines the wall friction velocity $u_{\tau}$. Starting from approximate initial values of these two quantities, a least squares method is used to improve the estimates of $y_{0}$ and $u_{\tau}$, by minimizing the least squares difference between the experimental profile and $u^{+}=y^{+}$, over the range $0 \leq y^{+}<20$, and $u^{+}=\kappa^{-1} \ln y^{+}+B$, at $y^{+}>35$. This procedure assumes that the horizontal mean velocity component $u$ is much greater than the mean vertical velocity component $v$ in the measured streamwise velocity $|\mathbf{u}|=\sqrt{u^{2}+v^{2}}$.

In each profile, the value of $y_{0}$ from the least squares fit is compared to the one measured using the stepper motor count. Over the 18 traverses, the difference between the two quantities was always less than 


\begin{tabular}{|c|c|c|c|c|c|}
\hline Configuration & Category & Tripping device & $x_{t}(\mathrm{~mm})$ & $\Delta x_{w}(\mathrm{~mm})$ & $R e_{x}$ \\
\hline 1 & a & No & 0 & 0 & 144256.6 \\
\hline 2 & a & No & 0 & 0 & 288493.2 \\
\hline 3 & a & No & 0 & 0 & 576986.3 \\
\hline \hline 4 & b & Wire & $400+1.25$ & 3.5 & 144256.6 \\
\hline 5 & b & Wire & $400+1.25$ & 3.5 & 288493.2 \\
\hline 6 & b & Wire & $400+1.25$ & 3.5 & 576986.3 \\
\hline \hline 7 & c & P40 & $228+10$ & 20 & 128219.4 \\
\hline 8 & c & P40 & $228+10$ & 20 & 256438.3 \\
\hline 9 & c & P60 & $228+140$ & 280 & 106849.3 \\
\hline 10 & c & P80 & $400+57.5$ & 115 & 128219.4 \\
\hline 11 & c & P80 & $400+57.5$ & 115 & 256438.3 \\
\hline \hline 12 & d & Granules & $400+24$ & 48 & 137835.6 \\
\hline 13 & d & Granules & $400+24$ & 48 & 275671.2 \\
\hline 14 & d & Granules & $400+24$ & 48 & 551342.5 \\
\hline 15 & d & Granules & $400+12$ & 24 & 141041.1 \\
\hline 16 & d & Granules & $400+12$ & 24 & 282082.2 \\
\hline 17 & d & Granules & $400+48$ & 96 & 131424.6 \\
\hline 18 & d & Granules & $400+48$ & 96 & 262849.3 \\
\hline
\end{tabular}

Table 1. Test cases.

$0.5 \mathrm{~mm}$, as stated in subsection II.C.

Having determined $y_{0}$, each experimental velocity profile is extended to the wall by adding the datum $(y, u)=(0,0)$ to the readings. The estimate for $u_{\tau}$ from the least squares method is then checked against its definition $u_{\tau}=\sqrt{\nu|\partial u / \partial y|_{y=0}}$ by determining the wall-normal gradient from $(y=0, u=0)$ and $\left(y=y_{0}, u=u_{0}\right)$. This check enhances the robustness of the data fit. In all traverses, the two $u_{\tau}$ estimates were found to be within an acceptable range from each other.

The experimental velocity profiles adjusted for $y_{0}$ are then examined to determine the boundary layer integral parameters ${ }^{5} \delta, \delta^{*}, \theta, H, u_{\tau}$, and $\operatorname{Re}_{\theta}$. The boundary layer thickness $\delta$ is taken as the wall-normal position at which $u(y)=0.99 u_{\infty}$. The displacement thickness $\delta^{*}$ and the momentum thickness $\theta$ are estimated by integrating the discrete experimental profile using Simpson's trapezoidal rule. $\delta^{*}$ and $\theta$ are then used to determine the shape factor $H=\delta^{*} / \theta$ and the momentum thickness based Reynolds number $R e_{\theta}=u_{\infty} \theta / \nu_{\infty}$.

Table 2 summarizes the boundary layer parameters $\left(\delta, \delta^{*}, \theta, H, u_{\tau}, R_{\theta}\right)$ from all 18 test cases of table 1. Configurations 1 to 3 are those from the wind tunnel floor natural boundary layer without any tripping device. In these three configurations, the boundary layer thickness $\delta$ is less than half of the target $20 \mathrm{~mm}$ thickness and the shape factor $H>2.5$ indicates that the boundary layer is essentially laminar. With a boundary layer trip, configurations 4 to 6 and 12 to 18 achieve a $\delta$ close to the $20 \mathrm{~mm}$ target. For these configurations, the shape factor $H \sim 1.5$ indicates a turbulent mean velocity profile.

\section{Flow analysis}

\section{V.A. Natural boundary layer}

Figure 2(a) shows the velocity profiles measured in the absence of any tripping device. These results correspond to configurations 1 to 3 in tables 1 and 2. At all three $R e_{x}$, the profile displays a near-constant velocity gradient close to the wall, which is characteristic of a laminar profile. The shape factor $H \geq 2.56$ is close to the Blasius solution for a laminar boundary layer of $H=2.59 . .^{5}$ The ratio $\delta / \delta^{*}$ is in the range $2.75 \leq \delta / \delta^{*} \leq 3.16$, which is also close to the Blasius analytical result of $\delta / \delta^{*}=2.8$. 


\begin{tabular}{|c|c|c|c|c|c|c|}
\hline Configuration & $\delta(\mathrm{mm})$ & $\delta^{*}(\mathrm{~mm})$ & $\theta(\mathrm{mm})$ & $H$ & $u_{\tau}(\mathrm{m} / \mathrm{s})$ & $R e_{\theta}$ \\
\hline 1 & 8.13 & 2.91 & 0.99 & 2.94 & 0.24 & 264.4 \\
\hline 2 & 6.53 & 2.20 & 0.77 & 2.85 & 0.32 & 411.4 \\
\hline 3 & 5.76 & 1.82 & 0.71 & 2.56 & 0.44 & 758.6 \\
\hline \hline 4 & 18.85 & 2.9 & 2.1 & 1.38 & 0.18 & 560.9 \\
\hline 5 & 18.85 & 2.62 & 1.81 & 1.45 & 0.36 & 967.0 \\
\hline 6 & 18.85 & 2.48 & 1.43 & 1.73 & 0.72 & 1527.9 \\
\hline \hline 7 & 8.76 & 3.01 & 1.14 & 2.64 & 0.21 & 304.5 \\
\hline 8 & 7.51 & 2.45 & 0.95 & 2.57 & 0.28 & 507.5 \\
\hline 10 & 8.25 & 3.10 & 1.00 & 3.10 & 0.22 & 267.1 \\
\hline \hline 11 & 7.00 & 2.58 & 0.83 & 3.08 & 0.29 & 443.4 \\
\hline 12 & 19.36 & 3.09 & 1.92 & 1.60 & 0.23 & 512.9 \\
\hline 13 & 19.14 & 3.38 & 2.22 & 1.56 & 0.41 & 1186.0 \\
\hline 14 & 18.56 & 3.08 & 2.08 & 1.48 & 0.76 & 2222.5 \\
\hline 15 & 18.73 & 3.03 & 1.97 & 1.54 & 0.22 & 526.2 \\
\hline 16 & 19.10 & 3.11 & 2.13 & 1.46 & 0.39 & 1137.9 \\
\hline 17 & 22.37 & 3.88 & 2.46 & 1.57 & 0.22 & 657.1 \\
\hline 18 & 23.16 & 4.01 & 2.69 & 1.50 & 0.40 & 1437.1 \\
\hline
\end{tabular}

Table 2. Measured boundary layer integral parameters from the test cases.

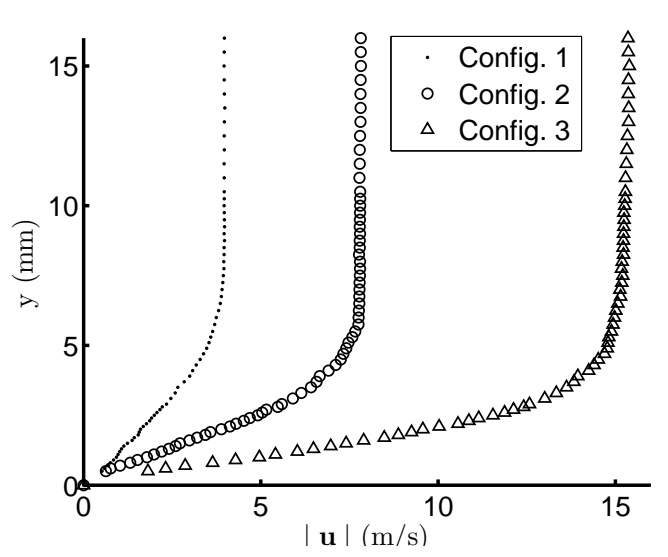

(a) Dimensional.



(b) Non-dimensional.

Figure 2. Velocity profiles on the wind tunnel floor without any boundary layer trip. 
Figure 2(b) shows the time-averaged velocity profiles normalized by the friction velocity $u_{\tau}$. A logarithmic scale is used on the abscissa to identify any portion of the velocity profile that displays a logarithmic velocity growth that would identify a transitional or turbulent boundary layer, resulting in a straight line on the logarithmic plot. No such trend is found in configurations 1 nor 3. Near the wall, the experimental data fits well the laminar sub-layer profile $u^{+}=y^{+}$, confirming that the time-averaged velocity near the wall grows at a constant gradient as in a fully laminar boundary layer. The velocity profiles from configurations 1,2 and 3 are therefore that of a laminar boundary layer with a maximum thickness of $8.13 \mathrm{~mm}$, as shown in table 2 . This differs from the target of developing a $20 \mathrm{~mm}$ thick turbulent boundary layer on the wind tunnel floor.

\section{V.B. Trip wire}

The results from configurations 4 to 6 in table 2 show that introducing a trip wire at the leading edge of the wind tunnel test section increases the boundary layer thickness to $\delta=18.85 \mathrm{~mm}$, close to the target of $\delta=20 \mathrm{~mm}$. This value is shown in table 2 to be independent from the free stream velocity. This implies that the boundary layer thickness is imposed by the wire wake and then increases very slowly in the downstream direction. The turbulent nature of the boundary layer, in this case, may be inferred from the values of $H$ between 1.38 ad 1.73, despite $H$ not decreasing at increasing Reynolds numbers, as it would be expected in a turbulent boundary layer. One possible justification for this is that, at a higher free-stream velocity $u_{\infty}$, the wire wake has less time to relax back into an equilibrium boundary layer before it reaches the measurement location $x_{p}$.

In a turbulent boundary layer, such as in a $1 / 7^{\text {th }}$ power law profile, $\delta / \delta^{*}=8.04$. With the trip wire, $6.5 \leq \delta / \delta^{*} \leq 7.6$, which is closer to the turbulent $\delta / \delta^{*}$ than the value reported in subsection V.A for the natural boundary layer.

\section{V.C. Sandpaper}

An alternative method to the trip wire to promote boundary layer transition is to use a sandpaper strip. The principle is to replace the confined vorticity shedding behind the wire by a uniform surface roughness distribution. The rationale is that, while a trip wire generates mainly spanwise vorticity, a roughness strip introduces also streamwise and flow-normal vorticity perturbations, aiding the development of a fully turbulent boundary layer downstream of it. The streamwise extent of the strip $\Delta x_{w}$ and its roughness height influence transition in the boundary layer, therefore sandpaper strips of different $\Delta x_{w}$ and grade were tested, with the objective of achieving a fully turbulent boundary layer.

Figure 3(a) shows the streamwise velocity profiles obtained at $R e_{x} \sim 0.128 \times 10^{6}$ using different strips. Configuration 1 in fig. 3(a) is the untripped boundary layer velocity profile from configuration 1 of table 1 that is re-plotted for reference. Figure 3(a) shows that the velocity profiles from configurations 7 and 10 overlap the untripped boundary layer profile of configuration 1 . These velocity traverses display the same near-wall constant gradient velocity profile featured in fig. 2(a). The effect of sandpapers in configurations 7 and 10 is to increase the boundary layer thickness by between $2 \%$ and $15 \%$ with respect to the wind tunnel natural boundary layer, as reported in table 2 . The shape factor with a boundary layer trip lies in the range $2.57 \leq H \leq 3.10$ and $2.66 \leq \delta / \delta^{*} \leq 3.10$. These ranges overlap those of the wind tunnel natural boundary layer and suggest that the $20 \mathrm{~mm}$ and $115 \mathrm{~mm} \Delta x_{w}$ sandpaper strips may have failed to induce transition.

The velocity measurements from the largest $\Delta x_{w}$ from configuration 9 give a profile that is different from all other configurations, as shown in figure $3(\mathrm{a})$. The $\Delta x_{w}=280 \mathrm{~mm}$ sandpaper strip gives a fuller profile close to the wall, followed by a near-constant velocity gradient that persists at $y=20 \mathrm{~mm}$. This streamwise velocity distribution could not be regressed on either a laminar nor a turbulent boundary layer, indicating that the $\Delta x_{w}=280 \mathrm{~mm}$ strip was too close to the measurement location $x_{p}$ for the flow to develop a profile with boundary layer type characteristics.

Figure 3(b) shows the velocity profiles from configurations 7 and 8 downsteam of the sandpaper trip in non-dimensional coordinates. The scale on the abscissa is logarithmic as in figure 2(b), to ease the identification of any portion of the velocity profile that features the logarithmic growth of a turbulent boundary layer, $u^{+}=\kappa^{-1} \ln y^{+}+B$. No such trend is clearly discernible in figure 3(b). Close to the wall, the laminar sub-layer analytical profile $u^{+}=y^{+}$is fitted to the normalized velocity measurements. This is shown to be a good fit to the experimental data, as in figure 2(b). This confirms the observations in figure 3 (a) of a constant gradient velocity profile close to the wall, extending approximately to $60 \%$ of the boundary layer thickness, which is a distinctive characteristic of a laminar boundary layer. This confirms that, over 




(a) Dimensional.

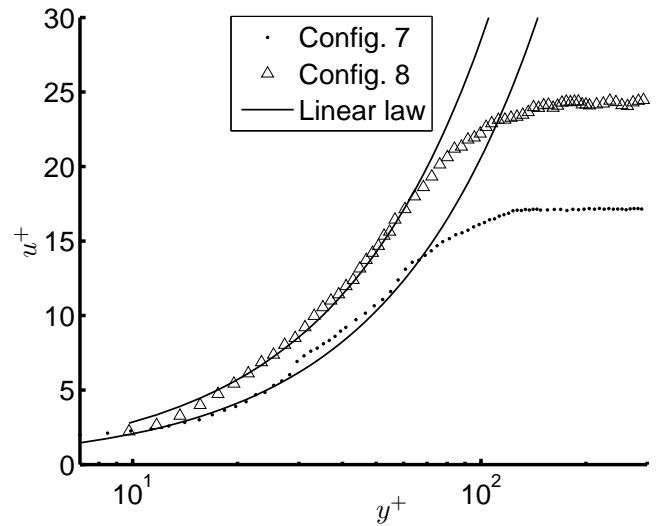

(b) Non-dimensional.

Figure 3. Velocity profiles for tripped boundary layer using a sandpaper strip at different Reynolds numbers.

the Reynolds number tested, $0.128 \times 10^{6} \leq R e_{x} \leq 0.256 \times 10^{6}$, the $\Delta x_{w}=20 \mathrm{~mm}$ and the $\Delta x_{w}=115 \mathrm{~mm}$ sandpaper strips are unable to induce transition and produce the desired turbulent boundary layer.

It is interesting to consider that although the $\Delta x_{w}=20 \mathrm{~mm}$ and the $\Delta x_{w}=115 \mathrm{~mm}$ sandpaper strips failed to develop a turbulent boundary layer of the required thickness, they were effective in altering the laminar boundary layer thickness. The use of tripping devices to control the thickness of laminar boundary layers is counter-intuitive and may have its use in applications where a thick laminar profile is desirable, for instance, to reduce the rate of heat transfer around thermally insulating panels.

\section{V.D. Silicon granules}

The third boundary layer trip technique tested is a strip of silicon granules. The principle is to create a strip of uniform roughness with a roughness height higher than that of the P40 coarsest sandpaper. The results in table 2 for configurations 12 to 18 show that this technique achieves more than a $200 \%$ increase in boundary layers thickness compared to the wind tunnel natural boundary layer. This can also be seen from figure 4(a), where the profiles from configurations 12 and 17 are plotted together with the reference profile of configuration 1. In figure 4(a), the boundary layer generated by the silicon granules appears fuller than the reference configuration 1 profile, featuring a more rapid increase in $|\mathbf{u}|$ velocity near the wall. These are characteristics typical of post-transition boundary layers. The shape factor obtained with the silicon granules strip lies in the range $1.48 \leq H \leq 1.60$ and $\delta / \delta^{*}$ is in the range $5.66 \leq \delta / \delta^{*} \leq 6.26$, confirming that a significant change in the velocity profile has taken place with respect to the wind tunnel natural boundary layer profile of fig. 2(a). With this boundary layer trip, $H$ and $\delta / \delta^{*}$ are closest to their reference values for a $1 / 7^{t h}$ power law of a turbulent boundary layer, $H=1.29$ and $\delta / \delta^{*}=8.04$, among the configurations tested. Configurations 12 to 14 in table 2 show that, by increasing $R e_{x}, H$ decreases towards the reference analytical value of $H=1.29$, suggesting that increasing $R e_{x}$ promotes the completion of the boundary layer transition over the wind tunnel floor, which is a desirable outcome towards achieving a fully turbulent $20 \mathrm{~mm}$ thick boundary layer. The effect of the inflow speed on the wall friction velocity is that doubling $u_{\infty}$ doubles $u_{\tau}$, as reported in table 2. This trend is closer to that for a turbulent boundary layer, for which a $1 / 7^{\text {th }}$ power law profile gives $u_{\tau} \propto u_{\infty}^{0.9}$, than that from a Blasius laminar profile, in which $u_{\tau} \propto u_{\infty}^{0.75}$.

Figures $4(\mathrm{~b})$ and $4(\mathrm{c})$ show respectively the velocity profiles obtained with $\Delta x_{w}=48 \mathrm{~mm}$ and $\Delta x_{w}=96$ $\mathrm{mm}$ wide silicon granule strips, in non-dimensional coordinates. The same logarithmic scale on the abscissa is used in figures 4(b) and 4(c) as in figures 2(b) and 3(b). In figures 4(b) and 4(c), the velocity profiles grow logarithmically over the range $30 \leq y^{+} \leq 70$, following the logarithmic law of the wall for turbulent boundary layers, $u^{+}=\kappa^{-1} \ln y^{+}+B$, which is shown in figures 4(b) and 4(c) by a solid line. This trend supports the observations in figure 4 (a) on the velocity profile shape being representative of a post-transitional boundary layer.

Figure 5(a) shows the Fourier analysis of the 32 seconds hot-wire velocity record taken at $y^{+}=65$ with the $\Delta x_{w}=48 \mathrm{~mm}$ narrow strip. $0.03 \mathrm{~Hz}$ narrow-band Power Spectral Levels of velocity are obtained by discrete Fourier transform of the discretized $|\mathbf{u}|$ velocity history with no anti-aliasing filter and no windowing. On the 


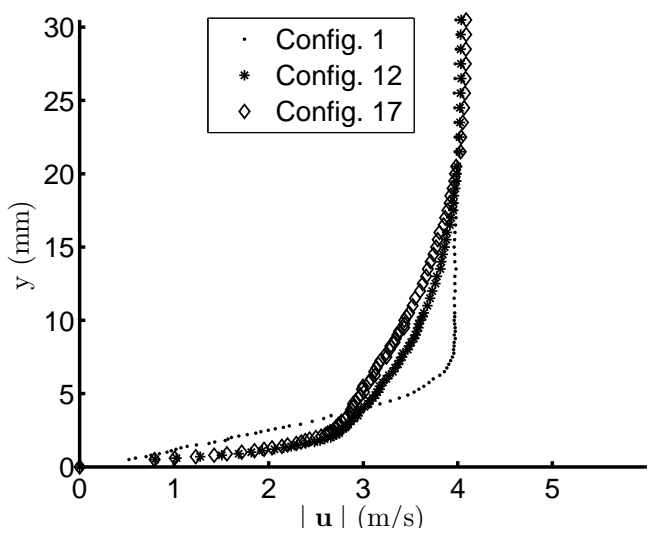

(a) Dimensional.



(b) Non-dimensional.

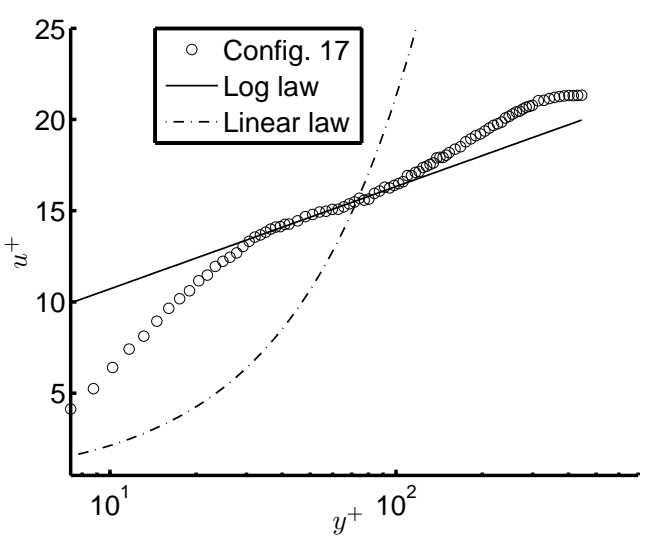

(c) Non-dimensional.

Figure 4. Velocity profiles for tripped boundary layer using a silicon granule strip at different Reynolds numbers. 
abscissa, the frequency range is $0 \leq f \leq 256 \mathrm{~Hz}, 256 \mathrm{~Hz}$ being the Nyquist frequency. The $0.03 \mathrm{~Hz}$ frequency resolution in figure 5(a) highlights a full broadband spectrum, the decay rate of which is comparable to the $-5 / 3$ exponential roll-of from fully developed isotropic turbulence spectra. This exponential decay is reported in the graph by a continuous line for reference.

The narrow-band Power Spectral Level distribution of figure 5(a) is rather noisy over the frequency range $50 \mathrm{~Hz}$ to $256 \mathrm{~Hz}$. To improve the signal to noise ratio in the results, the 32 seconds data was divided into 16 records of 2.28 seconds each with a $28 \%$ optimal overlap between contiguous segments, from which 0.44 $\mathrm{Hz}$ constant bandwidth Power Spectral Levels were obtained. Assuming the velocity fluctuations in the high frequency range are ergodic, as in fully developed turbulence, allowed to ensemble average the Power Spectral Levels over the 16 records. Figure 5(b) shows that this averaging has reduced considerably the scatter across the spectrum. The average of the first order upstream gradient of the Power Spectral Levels over the range $50 \mathrm{~Hz} \leq f \leq 256 \mathrm{~Hz}$ is -1.5535 . This gradient is shown by the dash-dot line in figure 5 (a). This compares reasonably well to the $-3 / 5$ gradient from fully developed turbulence spectra. This gradient is shown by the continuous line in figure 5 (b).

Reducing the strip width to $\Delta x_{w}=24$ while maintaining approximately the same free-stream velocity gives similar results, as shown in figures $5(\mathrm{c})$ and $5(\mathrm{~d})$. In this configuration, the gradient of the decay of turbulent velocity fluctuations is -1.8144 .

Figure 5(e) shows the narrow-band Power Spectral Level of velocity obtained from the $\Delta x_{w}=96 \mathrm{~mm}$ wider strip, taken at $y^{+}=68$. The sampling time and the acquisition frequency are the same as those of figure 5 (a), leading to the same $0 \mathrm{~Hz} \leq f \leq 256 \mathrm{~Hz}$ frequency range and $0.03 \mathrm{~Hz}$ frequency resolution. Whereas a full broadband spectrum is obtained like in figure $5(\mathrm{a})$, its decay rate over the frequency range $50 \mathrm{~Hz}$ to $256 \mathrm{~Hz}$ is about -1.4 , as indicated by the dash-dot line in the graph. This gradient is further away from the $-3 / 5$ decay rate from fully developed turbulence, shown by the solid line in figure $5(\mathrm{e})$, than in configurations 12 and 15.

To reduce the scatter in the Sound Pressure Level data, the 32 seconds dataset is divided in 16 segments of 2.396 seconds each, with an optimal overlap of $39 \%$ between contiguous segments. This overlap is larger than the one used for figure 5(b), giving $160.42 \mathrm{~Hz}$ constant bandwidth Power Spectral Density records that are ensemble averaged to give figure $5(\mathrm{f})$. In figure $5(\mathrm{f})$, the average of the first order upstream gradient of the Power Spectral Levels over the range $50 \mathrm{~Hz} \leq f \leq 256 \mathrm{~Hz}$ is -1.3738 . Similar results were obtained at the higher free-stream velocity of configuration 18, where the decay rate was found to be -1.1217 . This is further away from the theoretical decay rate of $-3 / 5$ of isotropic turbulence, indicating that using the $\Delta x_{w}=96 \mathrm{~mm}$ wider strip gives a boundary layer in which turbulence is further away from equilibrium than with the narrower strips, over the Reynolds number range $0.13 \times 10^{6} \leq R e_{x} \leq 0.26 \times 10^{6}$.

The configurations in which the decay rate of the velocity Power Spectral Level approximates better to that of a fully turbulent flow, $-5 / 3$, are configurations 12 and 15 , where the narrower $\Delta x_{w}=24 \mathrm{~mm}$ and $\Delta x_{w}=48 \mathrm{~mm}$ strips are used.

The highest Reynolds number at which the silicon granules trip was tested was $R e_{x}=0.55 \times 10^{6}$ of configuration 14. At this higher free-stream speed, the decay rate is further away from $-5 / 3$ than at $R e_{x}=0.276 \times 10^{6}$, indicating a boundary layer with turbulence further from being fully developed than at the lower speeds tested, probably due to the generation and shedding of more energetic structures that propagate downstream and decay more slowly as compared to the lower free stream velocity configurations.

\section{Boundary layer trip device selection}

A two-step ranking system is used to guide the selection of the boundary layer trip device that generates a flow closest to the target of a $20 \mathrm{~mm}$ thick fully turbulent boundary layer. In the first step, the performance of each device is computed as a compound non-dimensional error $\epsilon_{1}$ with respect to the integral boundary layer parameters $\left(\delta_{0}, H_{0}\right)$ of the target flow.

$$
\epsilon_{1}^{2}=c_{\delta_{0}}\left(\frac{\delta}{\delta_{0}}-1\right)^{2}+c_{H_{0}}\left(\frac{H}{H_{0}}-1\right)^{2}
$$

In equation $1, \delta_{0}=20 \mathrm{~mm}$ is the target boundary layer thickness and $H_{0}=1.4$ is the target shape factor for a fully developed turbulent boundary layer. The non-dimensional coefficients $c_{\delta_{0}}$ and $c_{H_{0}}$ in equation 1 are such that $c_{\delta_{0}}+c_{H_{0}}=1 . \quad c_{\delta_{0}}=2 / 3$ and $c_{H_{0}}=1 / 3$ are specified to give a higher weight to achieving the target boundary layer thickness of $20 \mathrm{~mm}$ with respect to matching the target shape factor $H_{0}$. While 




(a) Constant bandwidth Power Spectral Levels of velocity at $y^{+}=65$, Configuration 12 .

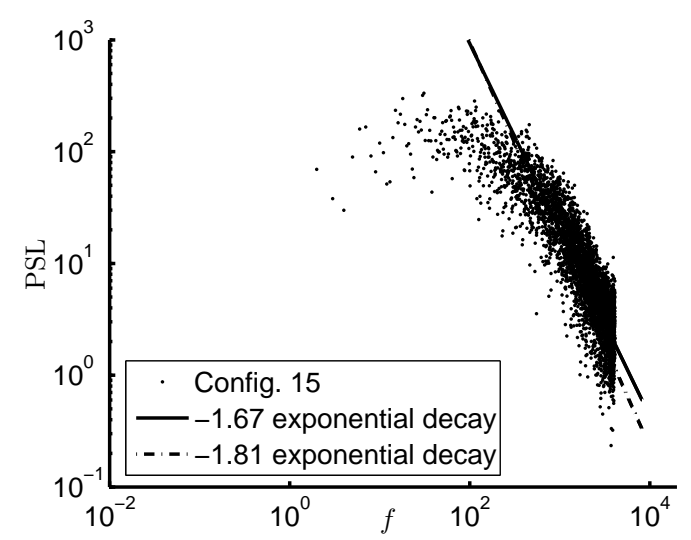

(c) Constant bandwidth Power Spectral Levels of velocity at $y^{+}=55.5$, Configuration 15 .

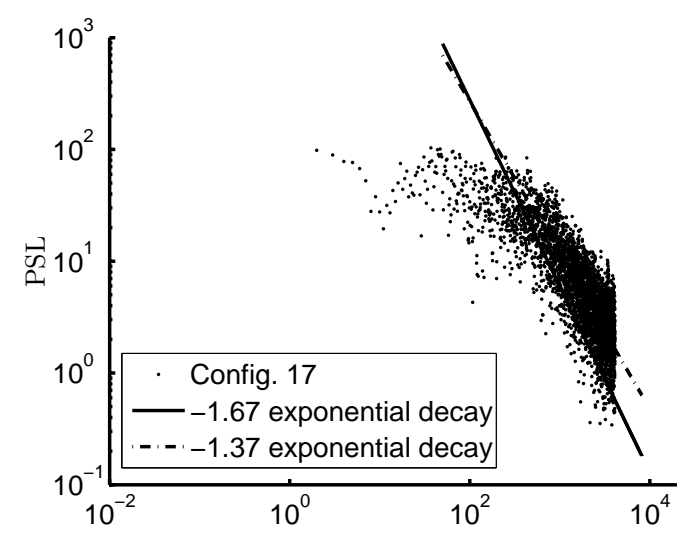

(e) Constant bandwidth Power Spectral Levels of velocity at $y^{+}=68$, Configuration 17 .

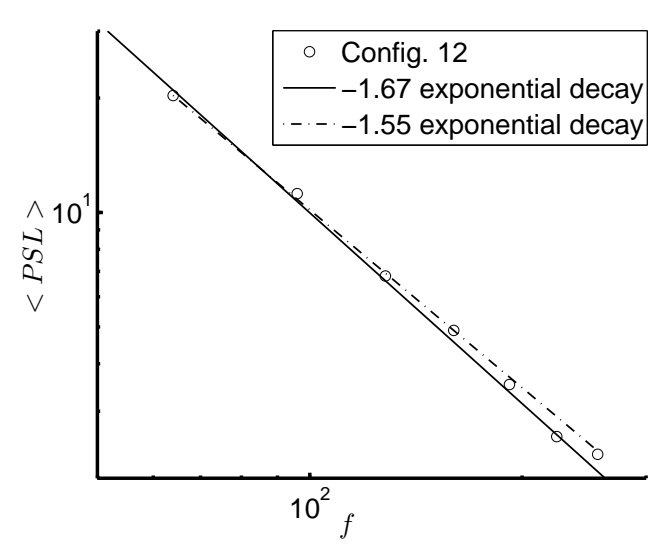

(b) Ensemble averaged Power Spectral Levels of velocity at $y^{+}=65$, Configuration 12 .



(d) Ensemble averaged Power Spectral Levels of velocity at $y^{+}=55.5$, Configuration 15 .

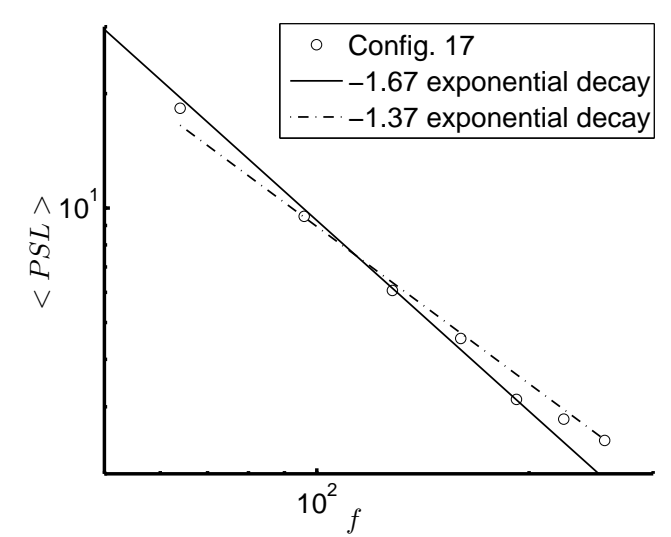

(f) Ensemble averaged Power Spectral Levels of velocity at $y^{+}=68$, Configuration 17 .

Figure 5. Velocity spectra from turbulent boundary layers tripped by silicon granules. 
$\delta_{0}=20 \mathrm{~mm}$ is a firm target, $H_{0}=1.4$ is used only as an indication of the turbulent state of the boundary layer. The turbulent state of the boundary layer will be re-checked in the second step of this two-step selection procedure. Therefore $H_{0}=1.4$ is taken as a soft target.

Computing $\epsilon_{1}^{2}$ generates a short-list from all configurations tested. This step is designed on purpose to be rather crude, so to involve the least amount of data processing that makes it scalable to an ensemble of trip device variants broader than the 18 configurations considered in table 1 . This step requires only time-averaged flow measurements as input, obtainable from simpler instrumentation, like a flattened head Pitot probe, than time-resolved data. Table 3 shows $\epsilon_{1}^{2}$ obtained from the 18 configurations tested.

\begin{tabular}{|c|c|c|c|}
\hline Configuration & $\frac{\delta-\delta_{0}}{\delta_{0}}$ & $\frac{H-H_{0}}{H_{0}}$ & $\epsilon_{1}^{2}$ \\
\hline 1 & $-59.35 \%$ & $+110.0 \%$ & $63.82 \%$ \\
\hline 2 & $-67.35 \%$ & $+103.6 \%$ & $66.02 \%$ \\
\hline 3 & $-71.20 \%$ & $+82.86 \%$ & $56.68 \%$ \\
\hline 4 & $-5.750 \%$ & $-1.428 \%$ & $0.227 \%$ \\
\hline 5 & $-5.750 \%$ & $+3.574 \%$ & $0.263 \%$ \\
\hline 6 & $-5.750 \%$ & $+23.57 \%$ & $2.072 \%$ \\
\hline 7 & $-56.20 \%$ & $+88.57 \%$ & $47.20 \%$ \\
\hline 8 & $-62.45 \%$ & $+83.57 \%$ & $49.28 \%$ \\
\hline 10 & $-58.75 \%$ & $+121.4 \%$ & $72.14 \%$ \\
\hline 11 & $-65.00 \%$ & $+120.0 \%$ & $76.17 \%$ \\
\hline 12 & $-3.200 \%$ & $+14.29 \%$ & $0.749 \%$ \\
\hline 13 & $-4.300 \%$ & $+11.43 \%$ & $0.559 \%$ \\
\hline 14 & $-7.200 \%$ & $+5.714 \%$ & $0.454 \%$ \\
\hline 15 & $-6.350 \%$ & $+10.00 \%$ & $0.602 \%$ \\
\hline 16 & $-4.500 \%$ & $+4.286 \%$ & $0.196 \%$ \\
\hline 17 & $+11.85 \%$ & $+12.14 \%$ & $1.427 \%$ \\
\hline 18 & $+15.80 \%$ & $+7.143 \%$ & $1.834 \%$ \\
\hline
\end{tabular}

Table 3. Compound non-dimensional difference from the target flow with different boundary layer trip devices.

The trip devices are short-listed such to minimize the compound error $\epsilon_{1}^{2}$. The short-list of the better performing trip devices is obtained by imposing $\epsilon_{1}^{2}<2.5 \%$. This threshold points towards configurations in which the profiles are either transitional or post-transitional. This is a pre-requisite to implement the second step in the two-step ranking system. From table 3, the configurations short-listed are the trip wire configurations $(4,5,6)$ and the granule strip configurations $(12,13,14,15,16,17,18)$.

The second step is designed to estimate the difference between the measured velocity profile and that from a zero pressure gradient fully turbulent boundary layer in the log-layer regions for the short-listed configurations. Hence, a second non-dimensional error $\epsilon_{2}$ is defined as:

$$
\epsilon_{2}^{2}=\frac{1}{N} \sum_{i=1}^{N}\left(\frac{u^{+}\left(y_{i}^{+}\right)-U_{\log }\left(y_{i}^{+}\right)}{U_{\log }\left(y_{i}^{+}\right)}\right)_{y_{i}^{+}>35}^{2}
$$

where $u^{+}\left(y_{i}^{+}\right)=\left|\mathbf{u}\left(y_{i}^{+}\right)\right| / u_{\tau}, U_{\log }\left(y_{i}^{+}\right)=\kappa^{-1} \ln y_{i}^{+}-B, N$ is the number of measured points at $y^{+}>35$ and $y_{i}^{+}$is the normalized wall-normal distance of the $i^{\text {th }}$ measurement point at $y^{+}>35$.

The $\epsilon_{2}^{2}$ percentage errors obtained from the 18 configurations tested are shown in table 4 . The best configuration with the lowest $\epsilon_{2}^{2}$ is configuration 12 , where a $\Delta x_{w}=48 \mathrm{~mm}$ wide granule strip is used at $R e_{x}=137835.6$.

\section{Concluding remarks}

The generation of a thick fully turbulent boundary layer at low velocity using different tripping devices has been addressed experimentally. For the present wind-tunnel, and in absence of a tripping device, the 


\begin{tabular}{|c|c|c|c|}
\hline Configuration & $\epsilon_{1}^{2}$ & $\epsilon_{2}^{2}$ & Ranking \\
\hline 4 & $0.227 \%$ & $\%$ & 8 \\
\hline 5 & $0.263 \%$ & $\%$ & 9 \\
\hline 6 & $2.072 \%$ & $\%$ & 10 \\
\hline \hline 12 & $0.749 \%$ & $0.076 \%$ & 1 \\
\hline 13 & $0.559 \%$ & $0.210 \%$ & 4 \\
\hline 14 & $0.454 \%$ & $0.286 \%$ & 7 \\
\hline 15 & $0.602 \%$ & $0.093 \%$ & 2 \\
\hline 16 & $0.196 \%$ & $0.247 \%$ & 6 \\
\hline 17 & $1.427 \%$ & $0.132 \%$ & 3 \\
\hline 18 & $1.834 \%$ & $0.245 \%$ & 5 \\
\hline
\end{tabular}

Table 4. Mean square percentage error of the logarithmic velocity profile for the short-listed configurations.

natural boundary layer is laminar over the test Reynolds number range. The maximum value of $\delta$, in this configuration, is equal to $8.13 \mathrm{~mm}$ leading to $R e_{\theta}=264.4$. The laminar state of the boundary layer is confirmed by the shape factor $H>2.5$ and the absence of a logarithmic growth in the normalized velocity profile.

Three different trip devices were tested. Applying a trip wire gives a significant growth in boundary layer thickness. However, the variation of $u^{+}$versus $y^{+}$in the resulting velocity profiles does not fit the logarithmic law of the wall. Furthermore, the variations of $\delta$ and $\theta$ with $u_{\infty}$ are very limited, making it difficult to adjust the set-up to obtain different boundary layer heights. Using a sandpaper strip allowed to improve the velocity profiles but the flow remained mainly laminar and the increase in boundary layer thickness was modest. With a sandpaper strip, the largest $\delta$ observed is equal to $8.76 \mathrm{~mm}$, leading to $R e_{\theta}=304.5$.

The idea of combining the effects of a high obstacle in the flow and that of a uniformly distributed roughness led to testing strips of silicon granules. With this trip device, $\delta \sim 20 \mathrm{~mm}$ and $H \sim 1.40$. The investigation of the normalized velocity and the statistical data has shown a good agreement with the logarithmic law for $u^{+}$and the exponential decay law for $\langle P S L\rangle$.

It has been shown that, even though wider silicon granule strips increase $\delta$, they cause a departure from equilibrium turbulence in the outer layer at the highest speed tested.

These results are of interest to the wind tunnel practitioner in the selection of an effective boundary layer trip for a low-speed wind tunnel test.

\section{Acknowledgements}

This research project has been supported by a Marie Curie Research Training Fellowship of the European Community's Sixth Framework Programme under contract MEST CT 2005 020301. The contributions of Mr. Paul Williams, Dr Yahya Akansu and Mr. Marco Grottaduarea are gratefully acknowledged.

\section{References}

\footnotetext{
${ }^{1}$ Casella, M., Castelluccio, V., and Marchese, P., "Measurements of the pressure fluctuations within an idealized door seal cavity in presence of flow," Technical report, Centro Ricerche Fiat, Orbassano, Italy, 2000.

${ }^{2}$ Ribaldone, E., Castelluccio, V., and Marchese, P., "Experimental and numerical study of flow excited cavities," Third International Congress in Sound and Vibration, Garmish, 2000.

${ }^{3}$ Grottadaurea, M. and Rona, A., "The role of the inflow momentum thickness in subsonic cylindrical cavity noise generation," 14 th International Congress on Sound and Vibration, No. 165, Cairns, Australia, July 2007.

${ }^{4}$ Rowley, C., Colonius, T., and Basu, A., "On Self-sustained Oscillations in Two-dimensional Compressible Flow Over Rectangular Cavities," J. Fluid Mechanics, Vol. 455, 2002, pp. 315-46.

${ }^{5}$ Calvert, J. R. and Farrar, R. A., An engineering data book, MacMillan Press Ltd, Houndmills, Basingstoke, Hampshire, UK, 1999.
} 